Opinion

\section{Mutability of Psychosis and Medications}

\author{
Peter F. Buckley
}

Virginia Commonwealth University School of Medicine, 1201 East Marshall St., 4th floor, Richmond, VA 23298, USA; Email: peter.buckley@vcuhealth.org.

It seems strange, even counterintuitive perhaps, that psychoses would exist separately each alongside symptom clusters representing perceptual, mood, and cognitive disturbances. And yet, since the dichotomous early thinking of Kraepelin who attempted on clinical and phenomenological grounds to parse out schizophrenia ("dementia praecox") from bipolar disorder ("manic depressive insanity"), the presence of psychotic symptoms with/without mood disturbances, and with/without cognitive impairment, has been the hallmark as to whether a psychosis be considered due to schizophrenia, due to a mood disorder, or due to some organic etiology ${ }^{[1-3]}$. The more recent convergence of genetic and neurobiological substrates across schizophrenia, mood disorders, and perhaps even more generally across psychiatric conditions belies both historical and current nosolgical perspectives on psychosis ${ }^{[4-7]}$. Recent genetics and molecular genetic studies-invariably of large sample sizes and ever increasingly with pooled U.S. and international data-in the aggregate suggest a greater convergence across major psychiatric disorders (schizophrenia, bipolar disorder, major depression) and perhaps even a broader constellation of conditions. Illustrative recent studies are now described. Ruderfer and colleagues conducted a genome-wide association study (GWAS) of some 54,000 patients with schizophrenia and bipolar disorder ${ }^{[8]}$. There was genetic overlap in identified loci, with bipolar disorder with psychotic features having a more distinct pattern. Howard and colleagues also conducted a 300,000 GWAS in depression that showed numbers of loci identified as well as convergence across schizophrenia, bipolar disorder, and depression ${ }^{[9]}$. Gandal and colleagues reported a major study of molecular neuropathology across several diagnoses. The transcriptome generated showed overlap across schizophrenia-bipolar, autism spectrum disorder-schizophrenia, autism spectrum disorder-bipolar disorder, and schizophreniamajor depression. Surprisingly, there was a low correlation between bipolar disorder and major depression. There were no associations with alcoholism, which stood alone in this analysis. These studies build upon the convergence between major psychiatric disorders in the seminal study that highlighted 108 significant gene associations! The description by a group of US research neuroscientists of three distinct, neurobiological subtypes of psychosis-so called "Biotypes"-is another provocative observation ${ }^{[10]}$. Psychosis might be a shared common pathway and/or phenotypic expression for different underlying biological substrates. This notion is already well established in cancer, epilepsy, and other brain disorders. Indeed, in cancer it is now even dictating treatment. Another important "biotype"-biomarker study by Moser and colleagues found convergence between bipolar and schizophrenia patients, both on clinical and imagining data ${ }^{[5]}$. The findings are complementary to the seminal study by elements and colleagues that describe the neurobiology of three distinct biotypes. 
How might "biotypes" inform treatment and how might treatment reflect "biotypes"? Clinically, it is not uncommon to see patients who received an initial diagnosis of schizophrenia who later on in their illness are "reclassified" as having a schizoaffective or bipolar. Did something change over time? Did they get "another" condition? Observation over time also provides potential insights on the impact of medications and how potentially there may alter the mutability of psychosis. Of course, longitudinal treatment and observational studies are hard to come by-though they often yield provocative findings. Decades ago, Courtney Harding and colleagues observed the virtual disappearance of schizophrenia some 30 years after discharge from hospital among patients who seemingly had the most recalcitrant of psychoses ${ }^{[11]}$. More recently, Wunderink and colleagues provocatively found that patients fared better earlier on in their illness when they stopped medication rather than being maintained on antipsychotic medications ${ }^{[12]}$. Meltzer also reports an intriguing case report of a patient whose illness was refractory over decades to multiple pharmacotherapies, yet late in life her illness appeared to remit with low doses of risperidone, and with noteworthy changes (gray matter increases) in brain structure on MRIs (Magnetic Resonance Imaging) ${ }^{[13]}$.

Historically, the concept of treatment response-and especially the allied concept of "treatment-refractory" schizophrenia-has been ill defined and overwhelmingly symptom-based ${ }^{[14]}$. Some studies of biological predictors of treatment response have pointed to structural brain abnormalities, to functional brain changes, and to perhaps more subtle neurochemical and peripheral markers that could-in varied instances and to widely variable reliability-inform treatment response. Indeed, Tim Crow originally proposed two distinct forms of schizophrenia-Type I and Type II-these being based upon different brain biology and consequently with different symptom profiles.

\section{REFERENCES}

1. Pearlson GD, Clementz BA, Sweeney JA, Keshavan MS, Tamminga CA. Does biology transcend the symptom-based boundaries of psychosis? Psychiatr Clin North Am. 2016; 39(2): 165-174.

2. Kircher T, Bröhl H, Meier F, Engelen J. Formal though disorders: from phenomenology to neurobiology. Lancet Psychiatry. 2018; 5(6): 515-526.

3. Hutchinson M, Stack JP, Buckley PF. Bipolar affective disorder prior to the onset of multiple sclerosis. Acta Neurol Scand. 1993; 6: 388-393.
On the other hand, when viewed through a more contemporary "neurobiological prism", the potential impact of medications upon different neurobiological substrates raises several interesting considerations. Firstly, there may be a neurobiological "match" for available medications, a possibility greatly overshadowed by the weak impact upon symptoms and marked heterogeneity of response. Second, future drugs might be "designed"/ developed toward distinct neurobiological substrates. Third, polypharmacy (that is presently random and "trial-and-error") might become rationale and personalized if each combination addressed distinct neurobiological perturbations. Fourth, the timing of introduction and use of different medications might be altered by a more rational appraisal of what neurobiological changes might occur over time. Presently, it appears that most interventionsincluding nonpharmacological ones-have some (and largely similar) impact in averting/forestalling psychosis when given to prodromal populations. At the other end of psychosis and over the course of illness, clozapine appears to be more effective than other drugs. And Meltzer's case report suggests that something changed biologically over time as exemplified by the later impact of a selective antipsychotic therapy ${ }^{[13]}$. Thus, understanding the biological trajectory and mutability of psychosis over time would be a powerful advance for our field.

\section{CONFLICTS OF INTEREST}

Buckley P.F. is Co-Investigator on the following federal grant: Bipolar-Schizophrenia Network for Intermediate Phenotypes II; National Institute of Mental Health, RO1.

4. Gandal MJ, Haney JR, Parikshak NN, Leppa V, Ramaswami G, Hartl C, et al. Shared molecular neuropathology across major psychiatric disorders parallels polygenic overlap. Science. 2018; 359(6376): 693-697.

5. Moser DA, Doucet GE, Lee WH, Frangou S, Krinsky $\mathrm{H}$, Leibu $\mathrm{E}$, et al. Multivariate associations among behavioral, clinical, and multimodal imaging phenotypes in patients with psychosis. JAMA Psychiatry. 2018; 75(4): 386-395.

6. Jauhar S, Nour MM, Veronese M, Rogdaki $\mathrm{M}$, Bonoldi I, Azis M, et al. A Test of the 
Transdiagnostic Dopamine Hypothesis of Psychosis Using Positron Emission Tomographic Imaging in Bipolar Affective Disorder and Schizophrenia. JAMA Psychiatry. 2017; 74(12): 1206-1213.

7. Schizophrenia Working Group of the Psychiatric Genomics Consortium. Biological insights from 108 schizophrenia-associated genetic loci. Nature. 2014; 511(7510): 421-427.

8. Ruderfer DM, Ripke S, McQuillin A, Boocock J, Stahl EA, Pavlides JM, et al. Genomic dissection of bipolar disorder and schizophrenia, including 28 subphenotypes. Cell. 2018; 173(7): 1705-1715.

9. Howard DM, Adams MJ, Shirali M, Clarke TK, Marioni RE, Davies G, et al. Genome-wide association study of depression phenotypes in UK Biobank identifies variants in excitatory synaptic pathways. Nat Commun. 2018; 9(1): 1470.

10. Clementz BA, Sweeney JA, Hamm JP, Ivleva El, Ethridge LE, Pearlson GD, et al. Identification of distinct psychosis biotypes using brain-based biomarkers. Am J Psychiatry. 2016; 173(4): 373-384.
11. Harding CM, Brooks GW, Ashikaga T, Strauss JS, Breier A. The Vermont longitudinal study of persons with severe mental illness, II: Longterm outcome of subjects who retrospectively met DSM-III criteria for schizophrenia. Am J Psychiatry. 1987; 144(6): 727-735.

12. Wunderink L, Nieboer RM, Wiersma D, Sytema S, Nienhuis FJ. Recovery in remitted first-episode psychosis at 7 years of follow-up of an early dose reduction/discontinuation or maintenance treatment strategy: long-term follow-up of a 2-year randomized clinical trial. JAMA Psychiatry. 2013; 70: 913-920

13. Meltzer HY, Sim MY, Anderson A, Cannistraci C, Jayathilake K, Share DB, et al. A within-subject consideration of the psychotic spectrum disorder concept in a patient in remission associated with cortical gray matter recovery. CNS Neurosci Ther. 2018; 24(7): 641-651.

14. Goff DC, Falkai P, Fleischhacker WW, Girgis RR, Kahn RM. The Long-Term Effects of Antipsychotic Medication on Clinical Course in Schizophrenia. Am J Psychiatry. 2017; 174: 840-849. 